\title{
Analysis and Comparison of Image Enhancement Methods in License Plate Recognition System
}

\author{
Yubing Dong ${ }^{1, a}$, Mingjing $\mathrm{Li}^{1, \mathrm{~b}}$, Ying Sun ${ }^{1}$ \\ ${ }^{1}$ College of Electronics and Information Engineering, Changchun University, \\ Changchun 130022, China \\ aemail: dyblbq@126.com, bemail: Imj5320510@163.com, 'email: 522929975@qq.com
}

Keywords: image enhancement; contrast enhancement; image smoothing; image sharpening

\begin{abstract}
In order to improve image quality in image processing, image enhancement method plays a very important role. This paper makes an exhaustive study of some enhancement methods such as contrast enhancement, image smoothing and image sharpening. And these methods are applied to License plate recognition. Various basic image enhancement techniques of License Plate Recognition are discussed and simulated with MATLAB. Through a lot of experiments, an improved image enhancement method is proposed by combining histogram equalization and median filtering. Comparing various image enhancement methods, the improved method can efficiently avoid interfere and enhance the contrast of image and obtain satisfying effects.
\end{abstract}

\section{Introduction}

With the progress of economy, science and technology, motor vehicles are becoming more popular. License plate recognition is particularly important for road information acquisition vehicle and vehicle management, etc. And license plate recognition is an important application and the core technology of computer vision and pattern recognition in the intelligent traffic field. License plate recognition system has been widely used. But under the influence of environmental factors such as bad weather, vehicle image will become blurred, due to the rain, snow, fog and noise. License plate recognition rate is serious decline. In order to improve the license plate recognition rate under the bad weather, image enhancement is to use a specific algorithm.

Image enhancement is located in the stage of image preprocessing. It plays an important role in the sense of low-grade process. The final enhancement results directly determine success or failure of the subsequent image processing in the sense of high-grade process. The aim of image enhancement is to process an image so that the result will be more suitable than the original image for a specific application. A method which is quite useful for enhancing an image may not necessarily be the best approach for enhancing another image. What is a good image? For human visual, the visual evaluation of image quality is a highly subjective process, and it is hard to standardize the definition of a good image; for machine perception, the evaluation task is easier, and a good image is one which gives the best machine recognition results. A certain amount of trial and error usually is required before a particular image enhancement approach is selected.

Image enhancement methods fall into two broad categories: spatial domain methods and frequency domain methods. Spatial domain methods are based on direct manipulation of pixel in an image, including of point operations and local operations. Frequency domain methods are based on modifying the Fourier transform of an image, including of DFT, Filter and IDFT. There are some enhancement techniques based on various combinations of methods from two categories.

This paper is organized as follows: section II presents various image enhancement methods. Section III gives simulation by MATLAB in License plate recognition, and section V presents subjective and objective evaluation image enhancement effects.

\section{Image Enhancement Methods}

According different effects of image enhancement, image enhancement method is divided into 
three classifications: contrast enhancement, image smoothing and image sharpening. Contrast enhancement methods mainly include: linear transform, non- linear transform, histogram equalization, histogram matching and local enhancement. Image smoothing methods mainly include: averaging mask, order-statistics filter and low pass filter. Image sharpening methods mainly include: derivatives and high pass filter. Each image enhancement method has its own advantages and disadvantages. Using different enhancement method, the image enhancement effects are different, so the applicable occasions are also different. This section introduces the principle of different image enhancement methods.

\section{Contrast Enhancement:}

The contrast enhancement can be limited in order to avoid amplifying the noise, which might be present in the image. The classification of contrast enhancement has direct gray level transformations, histogram processing and operations among images. The contrast enhancement operation is expressed by Eq.1, where $f(x, y)$ is the input image, $g(x, y)$ is the processed image, and $\mathrm{T}$ is an operator on $\mathrm{f}$ defined over some neighborhood of $(x, y)$.

$$
g(x, y)=T[f(x, y)]
$$

If the neighborhood is of size $1 \mathrm{x} 1$, g depends on the value of $\mathrm{f}$ at $(\mathrm{x}, \mathrm{y}), \mathrm{T}$ becomes:

$$
s=T(r)
$$

Where $r=f(x, y), s=g(x, y)$, then both s and $r$ are levels.

Histogram equalization is a method in image processing of contrast adjustment using the image's histogram. This method usually increases the global contrast of many images, especially when the usable data of the image is represented by close contrast values. Through this adjustment, the intensities can be better distributed on the histogram. The method is useful in images with backgrounds and foregrounds that are both bright or both dark. A key advantage of the method is that it is a fairly straightforward technique and an invertible operator. The transformation function is Eq.1. Where ${ }^{k=0,1 \ldots, L-1}, p\left(r_{j}\right)$ is the probability of occurrence of gray level in an image. Thus, an output image is obtained by mapping each pixel with level $r_{k}$ in the input image into a corresponding pixel with level $S_{k}$ in the output image.

$$
s_{k}=T\left(r_{k}\right)=\sum_{j=0}^{k} p\left(r_{j}\right)=\sum_{j=0}^{k} \frac{n_{j}}{n}
$$

\section{Image Smoothing:}

The aim of image smoothing is to diminish the effects of noise, spurious pixel values, missing pixel values etc. There are many different techniques for image smoothing; we will consider neighborhood averaging and edge-preserving smoothing. The median filter is a nonlinear digital filtering technique, often used to remove noise. For an even number of entries, there is more than one possible median, see median for more details. Where $W=\left(W_{m n}\right)\left(W_{m n}=1\right.$ or 0$)$ is expressed as a filter window, the center value of the filter window $(\mathrm{m}, \mathrm{n})$ is $(0,0)$, and $\left\{f(x, y)(x, y) \in I^{2}\right\}$ is expressed as the image gray value of each point. After $2 \mathrm{D}$ median filter, the output image is obtained by Eq.4.

$$
g(x, y)=\operatorname{Med}\{f(x, y)\}=\operatorname{Med}\left\{f(x+m, \quad y+n) \mid W_{m n}=1, \quad(x, y) \in I^{2}\right\}
$$

\section{Image Sharpening:}

The main aim in image sharpening is to highlight fine detail in the image, or to enhance detail that has been blurred. With image sharpening, we want to enhance the high-frequency components; this implies a spatial filter shape that has a high positive component at the centre. The Roberts cross operator is used in image processing and computer vision for edge detection. The kernels can be applied separately to the input image, to produce separate measurements of the gradient component in each orientation (call these Gx and Gy). The gradient magnitude is given by Eq. 5. In order to calculate faster, an approximate magnitude is computed by Eq. 6. The angle of orientation 
of the edge giving rise to the spatial gradient (relative to the pixel grid orientation) is given by Eq. 7.

$$
\begin{aligned}
& |G|=\sqrt{G_{x}^{2}+G_{y}^{2}} \\
& |G|=\left|G_{x}\right|+\left|G_{y}\right| \\
& \theta=\arctan \left(G_{y} / G_{x}\right)-3 \pi / 4
\end{aligned}
$$

\section{Experimental Results}

The original image is shown in Fig. 1, which is a picture of the license plate. Histogram equalization, median filter and Robert algorithm have been simulated on MATLAB. The effects of three image enhancement methods are shown in Fig. 2, Fig. 3 and Fig. 4. In order to improve clear of the image, histogram equalization is carried based on median filter. The enhanced image is obtained in Fig. 5, which has become clearer and more recognition of license plate.

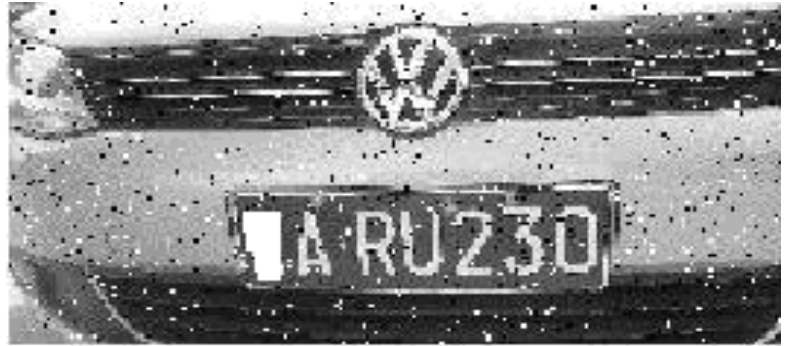

Fig.1 Original image

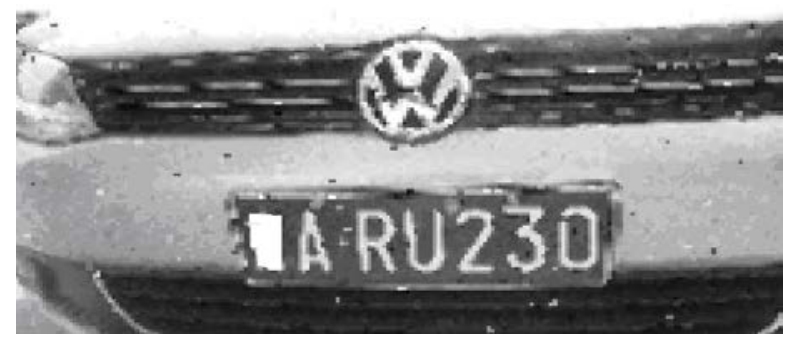

Fig.3 Enhanced image by median filter

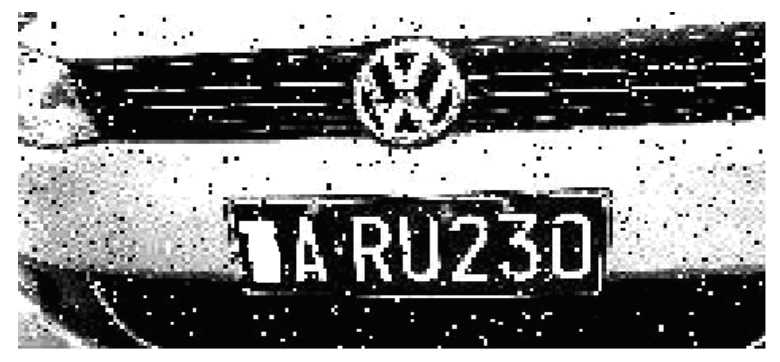

Fig.2 Enhanced image by histogram equalization

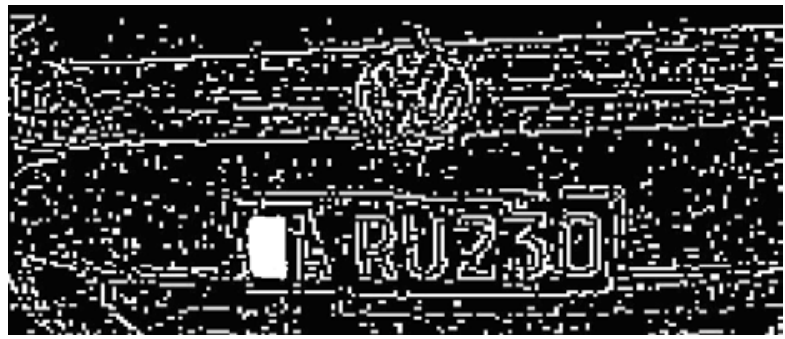

Fig.4 Enhanced image by Robert

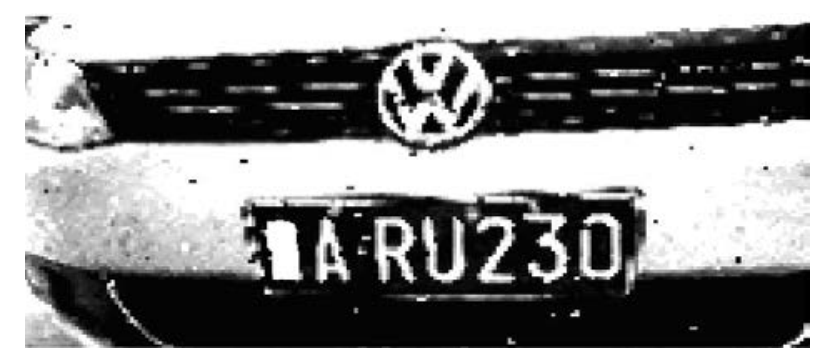

Fig.5. Enhanced image by histogram equalization based on median filter

\section{Conclusion}

In the paper, image enhancement based on histogram equalization, median filter, Robert cross operator and improved method by combining histogram equalization and median filtering, are deeply and systematically studied and compared. Image histogram equalization improves the contrast of the image. Two-dimensional image median filtering reduces high frequency. Image 
sharpening enhances the high frequency component of the image. To sum up, the different image enhancement methods are not the same as to the effect of image enhancement. As shown in the figures, the effect of this pair of license plate image histogram equalization based on median filter is better.

\section{Acknowledgement}

This work was financially supported by scientific research fund of Jilin provincial education department (2014, the number: 302), Project name is "Research on the key technology of portable target recognition system based on multi-sensors fusion”.

\section{References}

[1] Zhou Yunchuan, He Yongqiang, and Li Jitian, Infrared image enhancement method based on wavelet transformation and grayscale morphology, Laser \& Infrared, Vol. 41(2011), p. 683

[2] Mahdian B and Saic S, Periodic Properties of Interpolation and Their Application to Image Authentication[C]//Proc. of the 3rd International Symposium on Information Assurance and Security. Washington D. C., USA: IEEE Press, 2007, p. 439

[3] D. Jobson, Z. Rahman, and G. Woodell, A multiscale Retinex for bridging the gap between color images and the human observation of scenes, IEEE Trans Image Process, Vol. 6(1997), p.965.

[4] Meylan L, Susstrunk S, Hign Dynamic Range Image Rendering With a Retinex-Based Adaptive Filter, IEEE Transactions on Image Processing, Vol. 15(2006), p. 2820

[5] Wenjing Jia, Huaifeng Zhang, and Xiangjian He, Region-based license plate detection, Journal of Network and Computer Applications, Vol. 30(2007), p. 1324

[6] Kim S, Kim D, Ryu Y, and Kim G, A robust license-plate extraction method under complex image cinditions, Proceedings of the 16th international conference on pattern recognition, Vol. 3(2002), p. 216

[7] Huang T, Yang G, and Tang G, A fast two-dimensional median filtering algorithm, IEEE Transactions on Acoustics, Speech and Signal Processing, Vol. 27(2003), p. 13

[8] Bapeswara Rao V.V and Rao K.S, A new algorithm for real-time median filtering, IEEE Transactions on Acoustics, Speech and Signal Processing, Vol. 34(2003), p. 1674

[9] Md. Mehedi Masud, F. Keshtkar, and W. Gueaieb, Knowledge-based Image Segmentation using Swarm Intelligence Techniques, Int. J. Innovative Computing And Applications, Vol. 4( 2012), p.75 